\title{
Stereotactic Radiosurgery for Dural Arteriovenous Fistu- las Involving the Transverse-Sigmoid Sinus : A Single Center Experience and Review of the Literatures
}

\author{
Hong-Gyu Baek, ${ }^{1,2}$ Seong-Hyun Park, ${ }^{1,2}$ Ki-Su Park, ${ }^{1,2}$ Dong-Hun Kang, ${ }^{1-4}$ Jeong-Hyun Hwang, ${ }^{1,2}$ Sung-Kyoo Hwang ${ }^{1,2}$ \\ Department of Neurosurgery,' School of Medicine, Kyungpook National University, Daegu, Korea \\ Department of Neurosurgery, Kyungpook National University Hospital, Daegu, Korea \\ Department of Radiology, ${ }^{3}$ School of Medicine, Kyungpook National University, Daegu, Korea \\ Department of Radiology, ${ }^{4}$ Kyungpook National University Hospital, Daegu, Korea
}

Objective : We retrospectively assessed the efficacy of stereotactic radiosurgery (SRS) for dural arteriovenous fistulas (DAVFs) involving the transverse-sigmoid sinus and analyzed the angiographic and clinical results with our 8-year experience.

Methods : Nine patients with intracranial DAVFs involving the transverse-sigmoid sinus underwent SRS using a Gamma Knife ${ }^{\circledR}$ (Elekta Inc., Atlanta, GA, USA) between 2009 and 2016. Five patients underwent SRS for residual DAVFs after embolization and four patients were treated with SRS alone. The median target volume was $1.9 \mathrm{~cm}^{3}$ (range, 0.8-14.2) and the median radiation dose of the target was $17 \mathrm{~Gy}$ (range, 16-20). The median follow-up period was 37 months (range, 7-81).

Results : Pulsating tinnitus (33\%) was the most common symptom. DAVFs were completely obliterated in four patients (44\%) and subtotally obliterated in five (56\%). Six patients (67\%) showed complete recovery of symptoms or signs, and three (33\%) showed incomplete recovery. One patient experienced a recurrent seizure. Adverse radiation effects after SRS occurred in one patient (11\%). The total obliteration rates after SRS were $16.7 \%, 37.5 \%$, and $68.7 \%$ at 1,2 , and 3 years, respectively. The median interval from SRS to total obliteration of the fistula was 31 months (range, 12-38). The rates at which the symptoms started to improve were $40 \%$ at 1 month and $80 \%$ at 2 months after SRS. Symptoms started to improve at a median of 5 weeks after SRS (range, 3-21).

Conclusion : SRS with or without embolization is a safe and effective treatment to relieve symptoms and obliterate DAVFs on the transverse-sigmoid sinus.

Key Words : Central nervous system vascular malformations · Radiosurgery · Transverse sinuses.

\section{INTRODUCTION}

Dural arteriovenous fistulas (DAVFs) consist of a nidus of arteriovenous shunting within the dural mater and account for $10-15 \%$ of intracranial arteriovenous malformations ${ }^{3,99}$. Endovascular treatment has become the primary treatment for the majority of DAVFs, particularly those involving the cavernous sinus (CS) and transverse-sigmoid sinus, which are

\footnotetext{
- Received : November 9, 2018 •Revised : December 25, 2018 •Accepted : December 31, 2018

- Address for reprints : Seong-Hyun Park

Department of Neurosurgery, Kyungpook National University Hospital, School of Medicine, Kyungpook National University, 130 Dongdeok-ro, Jung-gu, Daegu 41944, Korea Tel : +82-53-200-5652, Fax : +82-53-423-0504, E-mail : nsdoctor@naver.com, ORCID : https://orcid.org/0000-0002-0982-5338
} 
difficult to access via a surgical approach ${ }^{6,10,11,15)}$. However, with endovascular treatment, it is often difficult to achieve successful embolization, and it has procedure- related complications. Remnant fistulas after embolization may lead to recruitment of the collateral vessels. Embolization for DAVFs involving the transverse-sigmoid sinus especially requires sacrifice of the major sinuses and often causes ischemic events. Recently, stereotactic radiosurgery (SRS) using a Gamma Knife (Elekta Inc., Atlanta, GA, USA) has become an alternative therapy for DAVFs and has been reported to lead to satisfactory results when treating DAVFs on the transverse-sigmoid sinuses ${ }^{6,14,16)}$. In the present study, we evaluated the clinical and angiographic outcomes of SRS using a Gamma Knife $^{\circledR}$ (Elekta Inc.) for DAVFs involving the transverse-sigmoid sinus and analyzed factors that were associated with improvement in symptoms and total obliteration of fistulas to demonstrate the efficacy of SRS for DAVFs involving the transverse-sigmoid sinus.

\section{MATERIALS AND METHODS}

\section{Study design}

We conducted a single-institution, retrospective study that was approved by the Institutional Review Board of Kyungpook National University Hospital (IRB No. KNUH 2014-03013-001). We evaluated the overall outcomes of nine consecutive patients with DAVFs involving the transverse-sigmoid sinus who underwent SRS with the Leksell Gamma Knife ${ }^{\circledR}$ (Elekta Inc.) at our institution between 2009 and 2016. The diagnosis of the DAVFs was confirmed by performing digital subtraction angiography (DSA). Angiography of the bilateral selective internal carotid artery (ICA), external carotid artery (ECA), and vertebral artery (VA) was performed in all patients to assess the feeding arteries, venous drainage, and site and size of the fistulas. SRS was recommended when surgical or endovascular access was difficult or there was incomplete obliteration after other treatments, and when there was a treatment-related risk. SRS alone was recommended for patients without symptoms or those with minor symptoms such as tinnitus or headache, and those without aggressive angiographic features such as cortical venous drainage (CVD). DAVFs that were suitable for analysis were identified using our SRS database and were verified on imaging studies con- ducted by a neuroradiologist. Outcome data were collected through an independent review of the patient's medical records and were analyzed by a neurosurgeon who had not participated in the patient's management.

\section{Classification of DAVFs}

DAVFs were classified according to the Borden et al. ${ }^{1}$ and Cognard et al.5) classification systems (Table 1). The Borden classification system is based on the site of venous drainage and the presence or absence of CVD. In type I, there is venous drainage directly into the dural venous sinus or meningeal vein (three patients, 33\%); in type II, there is venous drainage into the dural venous sinus with CVD (six patients, 67\%); and in type III, there is venous drainage directly into the subarachnoid veins (CVD only). Cognard's classification system is based on the direction of dural sinus drainage, the presence or absence of CVD, and venous outflow architecture. In type I, there is antegrade flow in the dural venous sinus (three patients, 33\%); in type IIa, there is retrograde flow in the dural venous sinus; in type IIb, there is antegrade flow and CVD in the dural venous sinus; in type IIa+IIb, there is retrograde flow and CVD in the dural venous sinus (six patients, 64\%); in type III, there is venous drainage directly into the subarachnoid veins (CVD only); in type IV, type III drainage occurs along with venous ectasias of the drained subarachnoid veins; and in type $\mathrm{V}$, there is direct drainage into the spinal perimed-

Table 1. Angiographic classification in nine patients with dural arteriovenous fistulas involving the transverse-sigmoid sinus treated with stereotactic radiosurgery

\begin{tabular}{ll}
\hline Finding & No. of patients \\
\hline Location & $4(44)$ \\
Right & $5(56)$ \\
Left & \\
Cortical vein drainage & $6(67)$ \\
With & $3(33)$ \\
Without & \\
Borden classification & $3(33)$ \\
1 & $6(67)$ \\
2 & \\
Congnard classification & $3(33)$ \\
I & $6(67)$ \\
\hline Values are presented llb number $(\%)$ &
\end{tabular}

Values are presented as number (\%) 
ullary veins.

\section{Stereotactic radiosurgery technique}

A Model G Leksell stereotactic frame was applied to all patients under local anesthesia. After attaching an imaging-compatible fiducial system to the frame, we performed high resolution magnetic resonance imaging (MRI) in all patients, followed by biplanar stereotactic angiography. Images were transferred to a computer workstation to plan dosing regimens. The DAVF nidus was outlined using optimized imaging integration of stereotactic angiography and MRI. All patients received an intravenous dose of 20-40 mg methylprednisolone after radiosurgery, and all were discharged from the hospital within 6-24 hours.

The SRS treatment parameters are shown in Table 2. The target volume was determined at the time treatment was planned, and the goal of the treatment was to envelop the three-dimensional nidus volume in a highly conformed and selective dose plan. The median target volume was $1.9 \mathrm{~cm}^{3}$ (range, 0.8-14.2). The median prescription dose delivered to the nidus margin was $17 \mathrm{~Gy}$ (range, 16-20). The median prescription isodose line was 50\% (range, 45-57\%). The maximum radiation dose ranged from 32 to $57 \mathrm{~Gy}$ (median, $36 \mathrm{~Gy}$ ).

\section{Follow-up examination}

The patients were instructed to undergo clinical follow-up examinations every 1 to 3 months after SRS and an imaging assessment (MRI, if possible) at 6, 12, 24, and 36 months. Neurological and otorhinolaryngological evaluations were performed at the time of imaging to assess the clinical outcomes. The beginning of symptom improvement was defined as the time at which the existing symptoms improved. Complete recovery was defined as return to normal function with clear evidence of clinical signs. MRI was performed to evaluate the response of the DAVF to radiation and detect any ad-

Table 2. Stereotactic radiosurgery treatment parameters

\begin{tabular}{lc}
\hline Factor & Value \\
\hline Median target volume $\left(\mathrm{cm}^{3}\right)$ & $1.9(0.8-14.2)$ \\
Median margin dose (Gy) & $17(16-20)$ \\
Median max dose (Gy) & $36(32-57)$ \\
Median isodose line (\%) & $50(45-57)$ \\
Median number of isocenters & $8(3-16)$ \\
\hline
\end{tabular}

Values are presented as number (range) verse radiation effects. After 2-3 years or if MRI and neurological evaluations demonstrated total obliteration, then DSA was requested. Total obliteration of DAVFs, as seen angiography, was defined as the elimination of fistulas and absence of an early draining vein. All patients had a minimum of 6 months of follow-up (median, 37 months; range, 7-81 months). Seven patients (78\%) had more than 2 years of follow-up.

\section{Statistical analysis}

For the statistical analysis we constructed Kaplan-Meier plots for the beginning of symptom improvement and total obliteration rates starting from the date of SRS and continuing until the last clinical and imaging follow-up examinations. The following variables were assessed : age, sex, symptom duration, lesion location and volume, angiographic classification, CVD, primary, residual, or recurrent lesions, prior embolization or surgery, history of hypertension, diabetes mellitus, cerebrovascular accident, cancer, head trauma, previous hemorrhage, initial presenting symptoms and signs, margin dose, maximal dose, isodose line, and number of isocenters. A probability value of less than 0.05 was set as significant. A univariate analysis was performed using the log-rank test (for categorical data) and the Cox proportional hazards model (for continuous data). Variables were compared using Fisher's ex-

Table 3. Characteristics in nine patients with dural arteriovenous fistulas involving the transverse-sigmoid sinus treated with stereotactic radiosurgery

\begin{tabular}{lc}
\hline Factor & Value \\
\hline Female & $5(56)$ \\
Median age in years & $63(41-78)$ \\
Median symptom duration in months & $1(0-56)$ \\
Median follow-up period in months & $37(7-81)$ \\
Treatment modality & \\
Radiosurgery alone & $4(44)$ \\
Embolization+radiosurgery & $5(56)$ \\
Initial signs and symptoms & \\
Pulsating tinnitus & $3(33)$ \\
Headache & $2(22)$ \\
Incidental finding & $2(22)$ \\
Seizure & $1(11)$ \\
Altered mental status & $1(11)$ \\
Hemorrhage at presentation & $1(11)$ \\
\hline
\end{tabular}

Values are presented as number (\%) or median (range) 
act test and the Mann-Whitney test. Standard statistical processing software (version 20.0, SPSS Inc., Chicago, IL, USA) was used.

\section{RESULTS}

\section{Patients}

There were five female patients and four male patients with a median age of 63 years (range, 41-78). Six DAVFs involving the transverse-sigmoid sinus were located on the left side (67\%), and three DAVFs were located on the right side (33\%). The pathogenesis of the DAVFs was predominantly idiopathic (in five patients, 56\%). Two patients (22\%) had lung cancer and breast cancer, respectively. One patient (11\%) had a history of head trauma that was treated with craniotomy. The other patient $(11 \%)$ experienced a transverse sinus thrombosis with an intracerebral hematoma. Five patients (56\%) underwent
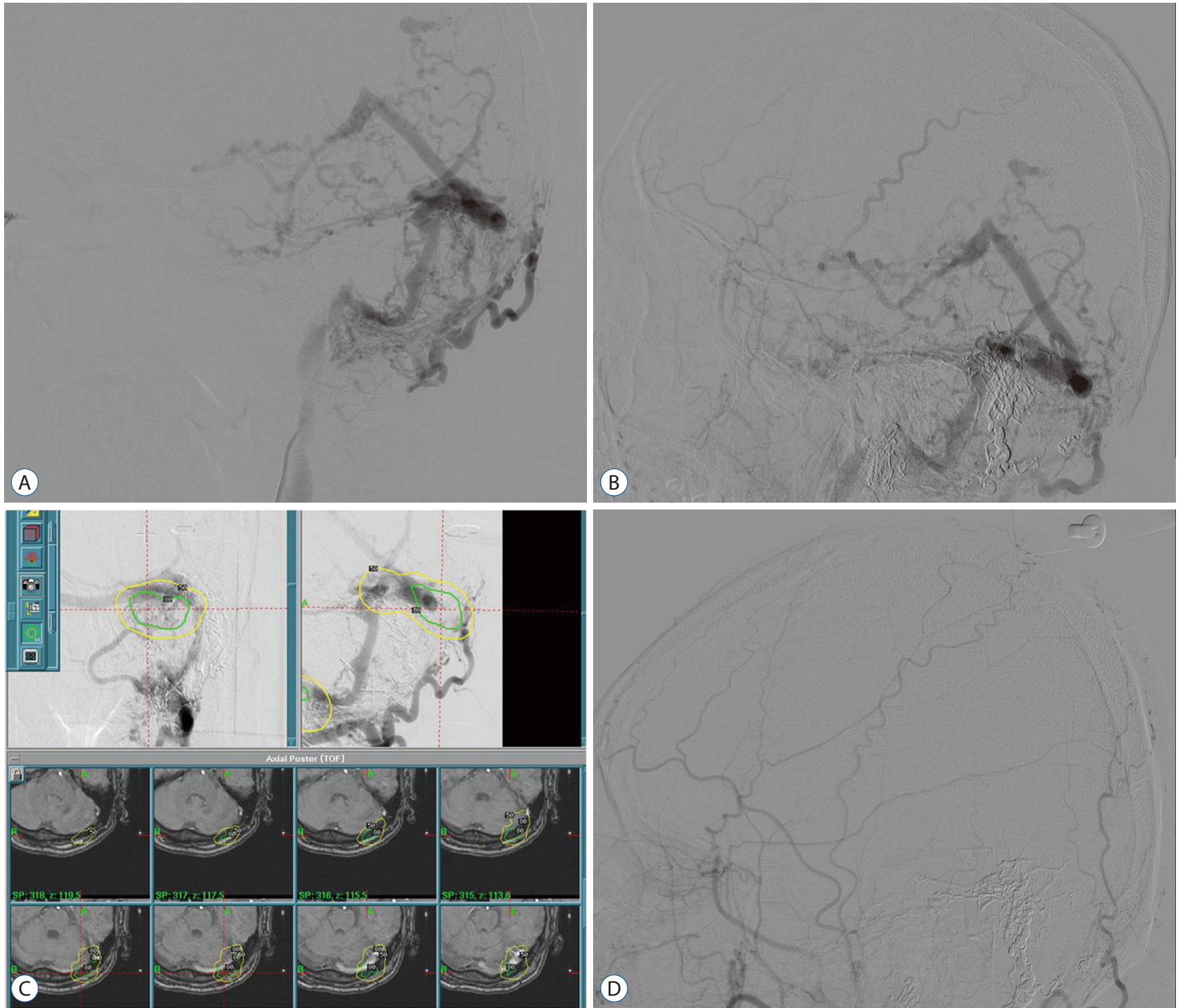

Fig. 1. A : Lateral angiographic view of the ECA for left DAVFs involving the transverse-sigmoid sinus before stereotactic radiosurgery. $B$ : Lateral angiographic view of the left ECA showing remnant fistulas with cortical venous drainage after transarterial embolization. C: Dose planning showing integration of stereotactic angiography and magnetic resonance images. The DAVF was treated with a radiation dose of $18 \mathrm{~Gy}$ at the $50 \%$ isodose line. D : A lateral angiographic view of the left ECA was obtained at 3 years after stereotactic radiosurgery, revealing complete obliteration of the fistulas. ECA : external carotid artery, DAVF : dural arteriovenous fistula. 
SRS for residual DAVFs after embolization and four (44\%) were treated with SRS alone. In five patients who underwent prior embolization, the median interval between embolization and SRS was 3 weeks (range, 1-13).

Seven patients (78\%) had neurological symptoms and/or signs at the time of SRS and two were asymptomatic. The median symptom duration was 1 month (range, 0-56). The demographic characteristics of the nine patients in this study are shown in Table 3. Pulsating tinnitus (33\%) was the most common symptom. Headaches occurred in two patients $(22 \%)$ and seizure occurred in one (11\%). Mental deterioration to a stuporous state was observed in one patient (11\%). Hemorrhage at presentation was detected in one patient (11\%).

\section{Radiological outcomes}

Obliteration of DAVFs after SRS was confirmed in the nine patients by using magnetic resonance angiography (MRA) or DSA. At the last neuroimaging follow-up examination, all patients had total or subtotal obliteration. DAVFs were totally obliterated in four patients (44\%) and subtotally obliterated in five (56\%). Fig. 1 demonstrates angiographic images of the obliterated DAVFs. Five patients whose neuroimaging results revealed subtotal obliteration are currently undergoing further follow-up examinations. Five patients (56\%) underwent

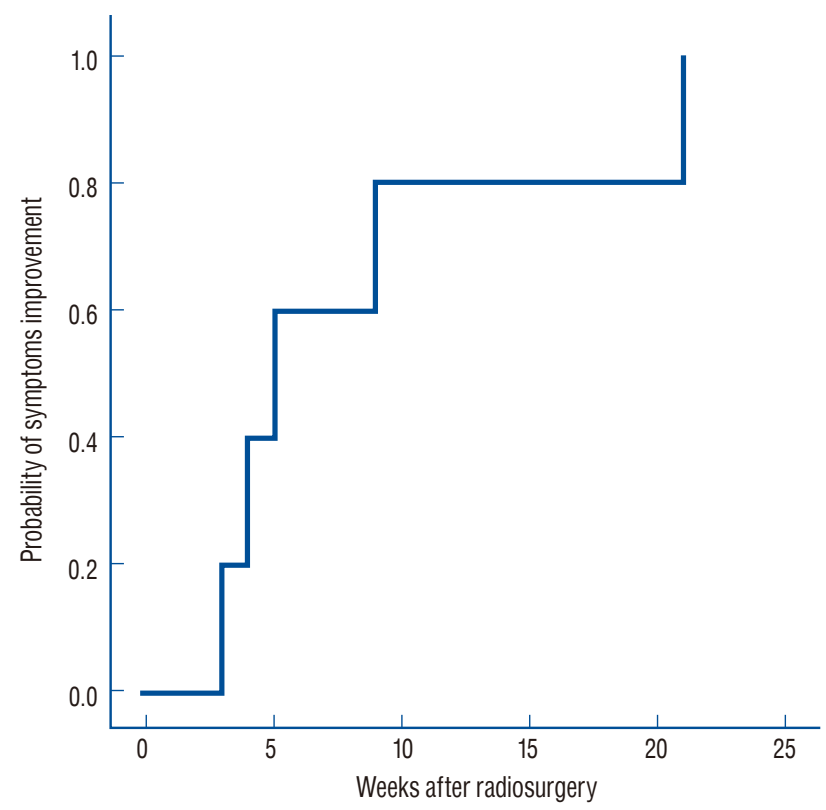

Fig. 2. Kaplan-Meier curve showing total obliteration of dural arteriovenous fistulas involving the transverse-sigmoid sinus after stereotactic radiosurgery.
DSA for evaluation of occlusion of the DAVFs and the other four (44\%) refused to undergo DSA and underwent MRA instead. Three of the five patients who underwent embolization before SRS had subtotal obliteration, and two had total obliteration after SRS. Two of the four patients who underwent SRS alone had total obliteration. None of these patients underwent any additional treatment after SRS during the followup period. One patient (11\%) had asymptomatic, new, highsignal intensity around the lesion on T2-weighted images at 68 months after SRS.

The total obliteration rates after SRS were $16.7 \%$ at 1 year, $37.5 \%$ at 2 years, and $68.7 \%$ at 3 years (Fig. 2). The median interval from SRS to total obliteration of the fistula was 31 months (range, 12-38). There were no significant differences in the obliteration rate according to the DAVF classification, presence of hemorrhage at presentation, CVD, combined therapy with embolization, volume, and radiation dose. DAVFs with and without CVD were completely obliterated in $67 \%$ and $0 \%$ of the patients, respectively ( $p=0.167)$. Residual DAVFs were completely obliterated after SRS with and without embolization in $40 \%$ and $50 \%$ of the patients, respectively $(p=0.643)$. In the univariate analysis, age $(p=0.022)$ was associated with the total obliteration of DAVFs. According to the multivariate analysis, none of these factors was associated

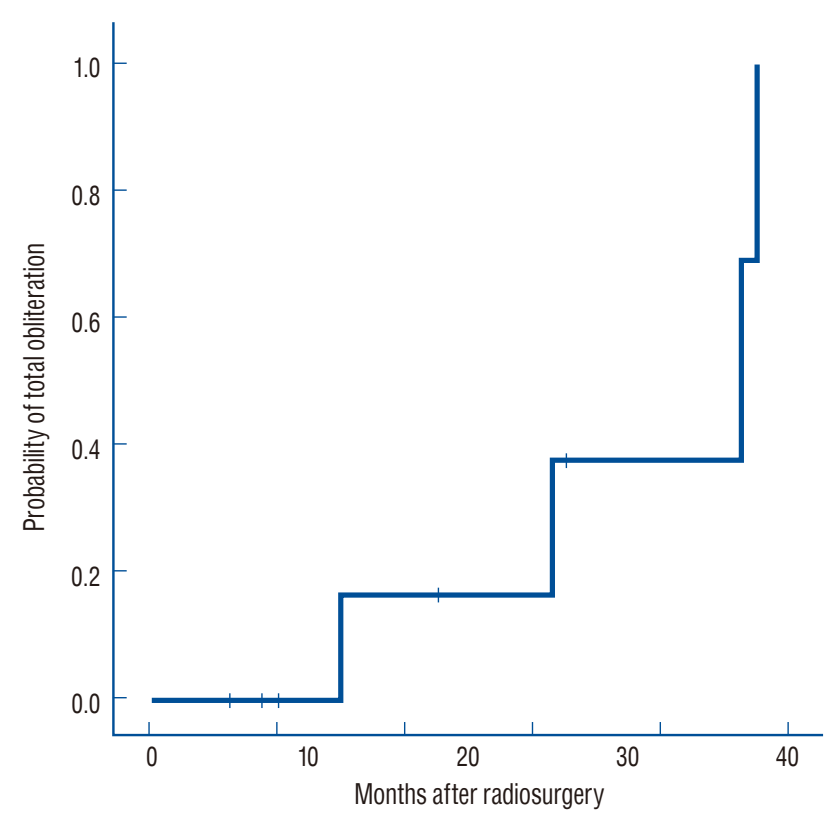

Fig. 3. Kaplan-Meier curve showing the improvement rate of neurological symptoms and signs after stereotactic radiosurgery. 
with the total obliteration of DAVFs.

\section{Overall clinical outcomes}

Most of the patient's symptoms, including tinnitus, headache, and altered mental status, improved after SRS. One patient had a recurrent seizure at 40 months after SRS. The Kaplan-Meier analysis demonstrated that the rates at which symptoms started to improve after SRS were $40 \%$ at 1 month and $80 \%$ at 2 months (Fig. 3). Symptoms started to improve at a median of 5 weeks after SRS (range, 3-21 weeks). In the univariate and multivariate analyses, no factor significantly affected improvement in neurological symptoms. At the last clinical follow-up examination, six patients (67\%) showed complete recovery of symptoms or signs and three (33\%) showed incomplete recovery. Two of the three patients with pulsating tinnitus showed complete recovery. Of them, one patient underwent SRS after embolization and the other was treated with SRS alone. No factors were associated with the complete recovery of neurological symptoms. Complete recovery from DAVFs, with and without CVD, was achieved in $50 \%$ and $100 \%$ of the patients, respectively ( $p=0.464)$. Complete recovery after SRS for DAVFs, with and without embolization, was achieved in $40 \%$ and $100 \%$ of the patients, respectively $(p=0.167)$.

\section{DISCUSSION}

We evaluated the clinical and angiographic outcomes of SRS for DAVFs involving the transverse-sigmoid sinus and analyzed the factors associated with improvements in symptoms and the total obliteration of fistulas. We found that SRS for DAVFs involving the transverse-sigmoid sinus led to an effective total obliteration rate, improvement in symptoms, and complete recovery, with a low risk of radiation-induced complications.

Currently, intracranial DAVFs are treated with microsurgery, endovascular embolization, SRS, and combination of these therapies. Endovascular embolization via a transvenous or transarterial approach has been considered the first-line option for patients with DAVFs involving the transverse-sigmoid sinus, which are often associated with venous hypertension, hemorrhage, or seizure $^{12}$. Although modern endovascular techniques have advanced greatly, embolization for DAVFs involving the transverse- sigmoid sinus is often difficult because sacrificing the major venous sinus and accidentally embolizing the distal venous system can have catastrophic consequences. It is also difficult for experienced neurointerventionalists to completely occlude DAVFs with endovascular embolization because of the complex angioarchitecture of these fistulas. Incomplete occlusion rather leads to recruitment of the collateral vessels and contributes to the persistent risk of hemorrhage. Recently, SRS has become an important additional or alternative treatment option for DAVFs, especially DAVFs involving the transverse-sigmoid sinus, when there is potential risk associated with surgical excision or embolization or when there are residual or recurrent lesions after surgical excision or embolization.

\section{Total obliteration after stereotactic radiosurgery}

It is always difficult to define multiple fistulas in the sinus wall on dose-planning angiographic views because DAVFs involving the transverse-sigmoid sinus have angiographically aggressive and complicated architecture with multiple arterial feeders and CVD. To effectively treat DAVFs, the neurosurgeon should include all multiple fistulas located along the sinus wall in the radiation target. In particular, selective angiography is important because multiple fistulas are supplied by various feeders from the dural branches of the ICA, ECA, and VA. In our cases, all DAVFs were supplied partly by the occipital artery. The areas of DAVFs, which were demonstrated with conventional angiography of the ECA and selective angiography of the occipital artery, were different. Care must be taken to notice the various feeders identified by selective angiography. Feeding arteries and drainage veins distal to the sinuses were not considered to be the nidus and were excluded from the treatment field.

Hanakita et al. ${ }^{7)}$ suggested that the targeted area should be limited to sites of arteriovenous communication. On angiographic views obtained during the early-to-late arterial phases, the sites at which the dural arteries drain directly into the dural sinus were located, and they were treated with a radiation dose of more than 20 Gy. Practically, it is difficult to only irradiate limited fistulas on the dural sinus wall when planning dosing regimens. Sometimes, a part of the surrounding venous sinus is inevitably irradiated. However, considering that the venous sinus is completely occluded with endovascular embolization via the transvenous approach, it does not seem to be clinically problematic that part of the venous sinus is in- 
cluded in the irradiation field. Unlike patients with arteriovenous malformations (AVMs), patients with DAVFs often refuse to undergo DSA to identify total obliteration because DAVFs show symptomatic improvement before they are completely occluded. In our patients, their symptoms already started to improve at 5 weeks after SRS, and DSA at 31 months after SRS confirmed that complete obliteration had occurred. Pollock et al. ${ }^{13)}$ and Kano et al. ${ }^{8)}$ reported that both MRI and DSA provide satisfactory evidence of the obliteration of AVM. However, it is difficult to detect early-draining veins and fistulas in the dural sinus wall with MRI. DSA is an important diagnostic tool for planning the next treatment options after a primary treatment for incomplete obliteration. We recommend DSA to angiographically confirm that DAVFs have been obliterated and determine additional therapy for the residual nidus, even if patients with complete recovery do not feel the need to undergo DSA. Previous reports of DAVFs involving the transverse-sigmoid sinus treated with SRS are summarized in Table 4. In a study on 16 patients with DAVFs involving the transverse-sigmoid sinus who were treated with SRS and embolization, Cifarelli et al. ${ }^{4}$ reported that the angiographic obliteration rate was $63 \%$. Pan et al. ${ }^{12)}$ reported that in 20 patients treated with SRS with or without surgery and/or embolization, complete obliteration occurred in $58 \%$ of the patients, subtotal obliteration occurred in $16 \%$, and partial obliteration occurred in $26 \%$. Friedman et al. ${ }^{6}$ reported that when SRS and embolization were combined to treat DAVFs of the transverse-sigmoid sinus, total or near-total obliteration was seen in 11 patients (65\%), and more than a 50\% reduction in six patients (35\%). The results of our study were similar. SRS with or without embolization provided a good obliteration rate for patients with DAVFs involving the transverse-sigmoid sinus. Although the number of patients and the statistical significance of the Kaplan-Meier curve were small, the rate of total obliteration would be useful in further studies, because there have not been comparable reports on only DAVFs involving the transverse-sigmoid sinus.

Yang et al. ${ }^{16)}$ reported that CS DAVFs were associated with higher rates of occlusion than transverse-sigmoid DAVFs, and suggested that in patients with DAVFs with more complex angioarchitecture, such as involving the transverse-sigmoid sinus, targeting the shunt is difficult. In addition, we hypothesize that a larger volume, recannalization of the embolized component, and different velocity of the shunting flow result in different outcomes in patients with transverse-sigmoid DAVFs than in those with CS DAVFs. Hanakita et al.7) reported that a lack of CVD, hemorrhage at presentation, a target volume less than $1.5 \mathrm{~cm}^{3}$, and Cognard type III or IV were associated with a higher obliteration rate. Age, sex, location, and prior therapy were not associated with obliteration. Chen et al. ${ }^{2)}$ demonstrated that the absence of CVD was associated with the obliteration of DAVFs. Our data showed that age, not the absence of CVD, was related to the obliteration of DAVFs, even though the statistical significance of our results was low

Table 4. Summary of previous reports of dural arteriovenous fistulas involving the transverse-sigmoid sinus treated with stereotactic radiosurgery

\begin{tabular}{|c|c|c|c|c|c|c|c|}
\hline Study & $\begin{array}{l}\text { No. of } \\
\text { Patients }\end{array}$ & Treatment & $\begin{array}{l}\text { Median } \\
\text { margin } \\
\text { dose (Gy) }\end{array}$ & $\begin{array}{c}\text { Symptom } \\
\text { improvement rate }\end{array}$ & Obliteration rate & $\begin{array}{l}\text { Radiation } \\
\text { induced } \\
\text { change }\end{array}$ & $\begin{array}{l}\text { Follow-up } \\
\text { (months) }\end{array}$ \\
\hline Pan et al. ${ }^{12)}(2002)$ & 20 & $\begin{array}{l}\text { SRS alone (11) } \\
\text { SRS+surgery and/or } \\
\text { Embo (9) }\end{array}$ & 16 & $\begin{array}{l}\text { 74\% complete } \\
26 \% \text { partial }\end{array}$ & $\begin{array}{l}58 \% \text { total } \\
16 \% \text { subtotal } \\
26 \% \text { partial }\end{array}$ & 0 & 19 \\
\hline Friedman et al. ${ }^{6)}$ (2001) & 23 & $\begin{array}{l}\text { SRS alone (3) } \\
\text { SRS+Embo (20) }\end{array}$ & 18 & $\begin{array}{l}96 \% \text { resolved or } \\
\text { improved }\end{array}$ & $65 \%$ total or near-total & 0 & 50 \\
\hline Cifarelli et al.') (2010) & 16 & SRS+Embo (16) & 21 & $\begin{array}{l}\text { 70\% good or excellent } \\
\text { (including CS) }\end{array}$ & $63 \%$ & 1 & 11.4 years \\
\hline Yang et al. ${ }^{16)}$ (2010) & 18 & $\begin{array}{l}\text { SRS with or without } \\
\text { Embo (18) }\end{array}$ & 21 & $\begin{array}{l}\text { 86\% improved } \\
\text { (including CS) }\end{array}$ & $\begin{array}{l}40 \% \text { CVD } \\
69 \% \text { no CVD }\end{array}$ & 0 & 45 \\
\hline Hanakita et al." (2012) & 9 & $\begin{array}{l}\text { SRS with or without } \\
\text { Embo (9) }\end{array}$ & 20 & $83 \%$ relieved & $55 \%$ & 0 & 33 \\
\hline Present series & 9 & $\begin{array}{l}\text { SRS alone (4) } \\
\text { SRS+Embo (5) }\end{array}$ & 17 & $\begin{array}{l}67 \% \text { complete } \\
33 \% \text { partial }\end{array}$ & $\begin{array}{l}44 \% \text { total } \\
56 \% \text { subtotal }\end{array}$ & 1 & 37 \\
\hline
\end{tabular}

SRS : stereotactic radiosurgery, Embo : embolization, CS : cavernous sinus, CVD : cortical venous drainage 
because of the small number of patients.

\section{Symptoms improvement and complete recovery after stereotactic radiosurgery}

In our study, no factors, including the total obliteration rate and prior embolization, were significantly associated with the rate of symptom improvement and complete recovery of neurological symptoms. Yang et al. ${ }^{16)}$ reported that CS DAVFs were associated with better symptom improvement than DAVFs of the transverse-sigmoid sinus. In studies on DAVFs involving transverse-sigmoid sinus that were treated by SRS, Friedman et al. $\left.{ }^{6}\right)$ reported a complete or partial symptom improvement rate of $96 \%$ and a total and nearly total obliteration rate of $65 \%$. Pan et al. ${ }^{12)}$ demonstrated a complete symptom improvement rate of $74 \%$, partial improvement rate of $26 \%$, and total and nearly total obliteration rate of $74 \%$. In both of these studies, the rate of improvement in symptoms was not consistent with the rate of total obliteration, as in our patients.

Asymptomatic, low-risk DAVFs can be observed and monitored. However, many patients with DAVFs exhibit a slow but progressive course; their symptoms at presentation may be progressive, and they may experience a seizure or hemorrhage, along with venous hypertension ${ }^{12}$. We considered active treatment for some patients with DAVFs, even those with a low hemorrhagic risk, if the DAVF was located on the transversesigmoid sinus. DAVFs with CVD have a significantly greater risk of hemorrhage as well as lower obliteration rate than do those without $\mathrm{CVD}^{2,4,7}$. Transverse-sigmoid sinus DAVFs are more likely to be accompanied by CVD and have more aggressive angiographic features than CS DAVFs. Therefore, we considered a more active treatment strategy because of the development of high-risk DAVFs in patients with the possibility of experiencing a hemorrhage or seizure. SRS with embolization was recommended for patients with aggressive angiographic features such as CVD. SRS alone was recommended in those with benign DAVFs or mild symptoms. Some patients were treated with SRS alone according to their condition or preference, and they had a good overall outcome, even though their DAVFs were included in the higher-risk category. This may be due to the rapid therapeutic response of DAVFs to SRS. The time until symptoms improved after SRS was relatively short, but not as much as that after embolization. In our results, CVD was not associated with improvement in symptoms or complete recovery. Active intervention with non-in- vasive SRS is helpful to resolve neurological symptoms. The angiographic architecture, severity of symptoms, and efficacy of treatment are important to determine the appropriate treatment for each patient.

\section{Limitations of the present study}

Although the present report on DAVFs is one of several SRS series to date that have focused on the location of DAVFs on the transverse-sigmoid sinus, the analysis of our results may have been limited by the small amount of data and the fact that this was a retrospective, single-center study. Multicenter trials should be performed to acquire sufficient data on SRS for DAVFs involving the transverse-sigmoid sinus to assess the efficacy of SRS.

\section{CONCLUSION}

Depending on the patient's condition and angiographic features of DAVFs, SRS with or without endovascular embolization can be a good therapeutic option for DAVFs involving the transverse-sigmoid sinus.

\section{CONFLICTS OF INTEREST}

No potential conflict of interest relevant to this article was reported.

\section{INFORMED CONSENT}

Informed consent was obtained from all individual participants included in this study.

\section{AUTHOR CONTRIBUTIONS}

\author{
Conceptualization : HGB, SHP \\ Data curation : KSP, DHK \\ Formal analysis : SHP \\ Funding acquisition : SHP \\ Methodology : JHH \\ Project administration : SHP
}




\author{
Visualization : SKH \\ Writing - original draft : HGB \\ Writing - review \& editing : SHP
}

\section{- Acknowledgements}

This research was supported by Kyungpook National University Research Fund, 2017.

\section{References}

1. Borden JA, Wu JK, Shucart WA : A proposed classification for spinal and cranial dural arteriovenous fistulous malformations and implications for treatment. J Neurosurg 82 : 166-179, 1995

2. Chen CJ, Lee CC, Ding D, Starke RM, Chivukula S, Yen CP, et al. : Stereotactic radiosurgery for intracranial dural arteriovenous fistulas: a systematic review. J Neurosurg 122 : 353-362, 2015

3. Chung SJ, Kim JS, Kim JC, Lee SK, Kwon SU, Lee MC, et al. : Intracranial dural arteriovenous fistulas: analysis of 60 patients. Cerebrovasc Dis $13: 79-88,2002$

4. Cifarelli CP, Kaptain G, Yen CP, Schlesinger D, Sheehan JP : Gamma knife radiosurgery for dural arteriovenous fistulas. Neurosurgery $67: 1230$ 1235; discussion 1235, 2010

5. Cognard C, Gobin YP, Pierot L, Bailly AL, Houdart E, Casasco A, et al. : Cerebral dural arteriovenous fistulas: clinical and angiographic correlation with a revised classification of venous drainage. Radiology 194 : 671-680, 1995

6. Friedman JA, Pollock BE, Nichols DA, Gorman DA, Foote RL, Stafford $S L$ : Results of combined stereotactic radiosurgery and transarterial embolization for dural arteriovenous fistulas of the transverse and sigmoid sinuses. J Neurosurg 94 : 886-891, 2001

7. Hanakita S, Koga T, Shin M, Shojima M, Igaki H, Saito N : Role of gamma knife surgery in the treatment of intracranial dural arteriovenous fistulas. J Neurosurg 117 Suppl : 158-163, 2012

8. Kano H, Kondziolka D, Flickinger JC, Yang HC, Flannery TJ, Awan NR, et al. : Stereotactic radiosurgery for arteriovenous malformations, part 3: outcome predictors and risks after repeat radiosurgery. J Neurosurg $116: 21-32,2012$

9. Kwon BJ, Han MH, Kang HS, Chang KH : MR imaging findings of intracranial dural arteriovenous fistulas: relations with venous drainage patterns. AJNR Am J Neuroradiol 26 : 2500-2507, 2005

10. Macdonald JHM, Millar JS, Barker CS : Endovascular treatment of cranial dural arteriovenous fistulae: a single-centre, 14-year experience and the impact of Onyx on local practise. Neuroradiology 52 : 387-395, 2010

11. Natarajan SK, Ghodke B, Kim LJ, Hallam DK, Britz GW, Sekhar LN : Multimodality treatment of intracranial dural arteriovenous fistulas in the Onyx era: a single center experience. World Neurosurg $73:$ 365-379, 2010

12. Pan DH, Chung WY, Guo WY, Wu HM, Liu KD, Shiau CY, et al. : Stereotactic radiosurgery for the treatment of dural arteriovenous fistulas involving the transverse-sigmoid sinus. J Neurosurg 96 : 823-829, 2002

13. Pollock BE, Kondziolka D, Flickinger JC, Patel AK, Bissonette DJ, Lunsford LD : Magnetic resonance imaging: an accurate method to evaluate arteriovenous malformations after stereotactic radiosurgery. J Neurosurg 85 : 1044-1049, 1996

14. Pollock BE, Nichols DA, Garrity JA, Gorman DA, Stafford SL : Stereotactic radiosurgery and particulate embolization for cavernous sinus dural arteriovenous fistulae. Neurosurgery 45 : 459-466; discussion 466467, 1999

15. van Rooij WJ, Sluzewski M : Curative embolization with Onyx of dural arteriovenous fistulas with cortical venous drainage. AJNR Am J Neuroradiol 31 : 1516-1520, 2010

16. Yang HC, Kano H, Kondziolka D, Niranjan A, Flickinger JC, Horowitz MB, et al. : Stereotactic radiosurgery with or without embolization for intracranial dural arteriovenous fistulas. Neurosurgery 67 : 1276-1283; discussion 1284-1285, 2010 